\title{
Controle da pressão arterial em idosas hipertensas em uma Unidade de Saúde da Família e fatores associados
}

\author{
Blood pressure control in hypertensive elderly women \\ attended in a Family Health Unit and associated factors
}

\author{
Maisa Bastos Moroz ${ }^{1}$, Ana Claudia Garabeli Cavalli Kluthcovsky \\ Marcelo Derbli Schafranski ${ }^{1}$
}

\begin{abstract}
Resumo
Introdução: A hipertensão arterial sistêmica é uma doença crônica de alta prevalência e com baixas taxas de controle. Este estudo objetivou analisar o controle da pressão arterial em idosas hipertensas acompanhadas em uma Unidade de Saúde da Família e os fatores associados. Métodos: Estudo transversal, com análise de dados de prontuários de 190 idosas hipertensas. Foram consideradas controladas as pacientes com nível de pressão arterial menor do que 140/90 mmHg. Para a análise estatística, calcularam-se a razão de prevalência e o intervalo de confiança de 95\% para a ocorrência de controle ou não da presão arterial, segundo a associação de alguns fatores. As análises multivariadas foram conduzidas por meio de regressão logística. Resultados: Das 190 pacientes em acompanhamento, 108 (56,8\%) apresentavam a pressão arterial controlada. Na análise de regressão logística, as variáveis possuir mais de 70 anos $(p=0,033)$ e residir na área mais afastada da unidade de saúde $(p=0,002)$ estiveram associadas ao não controle da pressão arterial. Conclusão: Embora o percentual de controle da pressão arterial seja elevado na população estudada quando comparado com dados da literatura, medidas devem ser implantadas para o aumento desse percentual, para evitar futuras complicações.
\end{abstract}

Palavras-chave: hipertensão; controle; idoso; Centros de Saúde.

\begin{abstract}
Introduction: Hypertension is a chronic disease with high prevalence and low control rates. This study aimed to analyze the blood pressure control in hypertensive elderly followed up in a Family Health Unit and its associated factors. Methods: Cross-sectional study, with patient records analysis of 190 hypertensive elderly women. Patients' blood pressure was considered to be under control if lower than $140 / 90 \mathrm{mmHg}$. For statistical analysis, we calculated the prevalence ratio and $95 \%$ confidence interval for controlled and not controled blood pressure, according to the association of some factors. Multivariate analyzes were conducted using logistic regression. Results: Of the 190 patients followed up, 108 (56.8\%) had controlled blood pressure. In logistic regression analysis, the variables of being older than 70 years $(p=0.03)$ and reside in the area furthest from the facility $(p=0.002)$ were associated with uncontrolled blood pressure. Conclusion: Although the control rate in this population is high when compared with literature, measurements should be implemented to increase this rate and avoid future complications.
\end{abstract}

Keywords: hypertension; control; elderly; Health Centers.

'Departamento de Medicina, Universidade Estadual de Ponta Grossa - UEPG - Ponta Grossa (PR), Brasil.

Trabalho realizado em uma Unidade de Sáude da Família de um município da região Sul do Brasil e na Universidade Estadual de Ponta Grossa (UEPG) - Ponta Grossa (PR), Brasil.

Endereço para correspondência: Ana Claudia Garabeli Cavalli Kluthcovsky - Av. Coronel Carlos Cavalcante, 4748 - Uvaranas - CEP: 84030-900 - Ponta Grossa (PR), Brasil-Email: anafabio2009@gmail.com

Fonte de financiamento: nenhuma.

Conflito de interesses: nada a declarar. 


\section{INTRODUÇÃO}

A hipertensão arterial sistêmica (HAS) é uma doença crônica de alta prevalência e com baixas taxas de controle, sendo um grande problema de saúde pública ${ }^{1,2}$. É a condição mais comum vista em cuidados primários de saúde e pode levar ao infarto do miocárdio, acidente vascular cerebral, insuficiência renal e morte se não for detectada precocemente e tratada adequadamente ${ }^{3}$. Estima-se que a prevalência da HAS seja em torno de $30 \%$ tanto para a população brasileira quanto para a população mundial ${ }^{4}$, percentual que aumenta proporcionalmente com o aumento da idade ${ }^{2}$.

Em revisão sobre o panorama do controle da hipertensão arterial no Brasil, nos estudos de base populacional o índice varia de $10 \%$ a 57,6\%. Para a Estratégia Saúde da Família (ESF), a frequência de controle variou de $30 \%$ a $53,9 \%$, sendo que, para pacientes idosos, foram observados piores índices, variando de $27 \%$ a $44,6 \%{ }^{4}$.

$\mathrm{O}$ controle da pressão arterial (PA) é imprescindível para a redução da morbimortalidade associada à doença e depende não só da adesão dos pacientes, mas também dos profissionais de Saúde e das ações institucionais ${ }^{5}$. Entre os fatores para o não controle da PA relacionados aos pacientes estão a dificuldade de acesso aos serviços de saúde, a idade avançada, a obesidade e a não adesão ao tratamento. Causas relacionadas aos profissionais incluem falta de conhecimento ou não adesão aos protocolos, preocupação com efeitos colaterais dos medicamentos e relutância em tratar uma condição "assintomática".

Diante desse contexto, a ESF, pautada pelos princípios do Sistema Único de Saúde, visa a expansão, qualificação e consolidação da Atenção Básica, por favorecer uma reorientação do processo de trabalho ${ }^{7}$. Nessa proposta, o manejo adequado da hipertensão arterial adquire caráter prioritário ${ }^{8}$.

Considerando a importância do controle da PA para reduzir a morbimortalidade e o escasso número de publicações sobre o tema, este trabalho teve como objetivo analisar o controle da PA em idosas hipertensas acompanhadas em uma Unidade de Saúde da Família (USF) e determinar os possíveis fatores associados.

\section{METODOLOGIA}

Trata-se de um estudo transversal de abordagem quantitativa, realizado com dados obtidos de prontuários de mulheres idosas hipertensas acompanhadas em uma USF. Essas pacientes foram selecionadas a partir do cadastro dos 840 portadores de hipertensão arterial da unidade de saúde, pelos critérios sexo e idade.

A USF onde o estudo foi realizado conta com duas equipes de Saúde da Família (responsáveis pelo atendimento de duas áreas, aqui chamadas de A e B), que foram implantadas no ano de 2001, em uma cidade de médio porte localizada na Região Sul do Brasil referência na região para a área da saúde e educação.
Primeiramente foram analisados todos os 840 prontuários dos pacientes hipertensos cadastrados na USF. Os critérios de inclusão foram: mulher hipertensa cadastrada na USF, ter 60 anos de idade ou mais e fazer uso de um ou mais medicamentos anti-hipertensivos. Considerando esses critérios e três prontuários excluídos por grande quantidade de dados ilegíveis ou incompletos, foram selecionados 251 prontuários. A seguir foi realizada uma nova seleção, dos prontuários das pacientes em acompanhamento na unidade, ou seja, daquelas que compareceram a pelo menos uma consulta médica no último ano, contado a partir da data da coleta de dados ${ }^{9}$. Dos 251 prontuários, 190 (75,7\%) eram de pacientes classificadas como em acompanhamento, cujos dados foram analisados neste estudo.

Para a classificação das 190 pacientes em controladas ou não controladas foi utilizado o valor da medida da PA registrada no prontuário na última consulta médica. Foram classificadas como controladas aquelas com valores menores de $140 \mathrm{mmHg}$ de pressão arterial sistólica (PAS) e de $90 \mathrm{mmHg}$ de pressão arterial diastólica (PAD). Há grande controvérsia na literatura em relação ao ponto de corte para o qual a PA pode ser considerada controlada na população idosa, principalmente nos idosos com idade mais avançada (acima dos 80 anos). Estudos recentes sugerem uma meta de controle mais flexível (abaixo de $150 \mathrm{mmHg}$ para a PAS e de $90 \mathrm{mmHg}$ para a PAD) para esta faixa etária, por evidenciarem que o controle mais rigoroso pode não ser significativo na redução das complicações associadas à doença ${ }^{3,10-12}$. Contudo, neste estudo adotou-se o preconizado pela VI Diretrizes Brasileiras de Hipertensão para o controde da PA em idosos, sendo o mesmo critério utilizado pela maior parte dos estudos brasileiros publicados sobre o tema ${ }^{2,4}$.

A coleta de dados ocorreu entre agosto de 2013 e janeiro de 2014, por meio da análise dos prontuários. Utilizou-se um formulário contendo dados sociodemográficos, informações clínicas e do tratamento, elaborado a partir de dados da literatura ${ }^{13,14}$, além de informações contidas na ficha $\mathrm{A}$ do Sistema de Informação da Atenção Básica.

As variáveis sociodemográficas analisadas foram: idade, alfabetização, ter ou não plano de saúde, número de habitantes na moradia, trabalho remunerado (ou pensão ou aposentadoria) e local de residência em relação à área da Saúde da Família pela qual era acompanhada (área $\mathrm{A}$ mais próxima e área $\mathrm{B}$ mais afastada da USF).

Para as variáveis clínicas e de tratamento foram analisados: peso, altura, valores da PA, tempo de acompanhamento na unidade, determinado pela primeira consulta registrada no prontuário, número de consultas médicas no último ano, medicamentos em uso, presença de comorbidades (diabetes, dislipidemia, obesidade, doenças cardiovasculares, insuficiência cardíaca, acidente vascular cerebral e doença renal crônica). 
Não foi possível obter os dados sobre sedentarismo e tabagismo devido ao escasso registro dessas informações nos prontuários, o que também ocorreu com o registro de histórico familiar de doença coronariana e hipertrofia ventricular esquerda.

A obesidade foi classificada pelo Índice de Massa Corporal (IMC) de acordo com a seguinte fórmula: [IMC = (peso em $\mathrm{kg}) /$ (altura em metros) $)^{2}$. Foram classificadas como obesas as pacientes com IMC $\geq 30,0 \mathrm{~kg} / \mathrm{m}^{15}$.

Para apresentar as características das pacientes e os valores da PA foram realizadas estatísticas descritivas. Para análise estatística, a comparação entre as médias das pressões sistólica e diastólica entre as pacientes com PA controlada e não controlada foram realizadas pelo Teste de Mann Whitney após análise de histogramas e Teste de Kolmogorov-Smirnov para verificação de gaussianidade. Calculou-se a razão de prevalência e o intervalo de confiança de $95 \%$ para o controle ou não da $\mathrm{PA}$, segundo a associação das variáveis sociodemográficas, clínicas, de tratamento e comorbidades. As análises multivariadas foram conduzidas por meio de regressão logística, para identificar as possíveis variáveis associadas. Foram incluídas no modelo as variáveis com valor de $\mathrm{p}<0,20$ na análise bivariada, calculando-se odds ratio e respectivos intervalos de confiança de 95\%. Os dados foram processados no banco de dados Excel 2010. A análise estatística foi realizada com auxílio do programa Statistical Package for Social Science, versão 16.0. O nível de significância utilizado foi de $5 \%$. O protocolo de pesquisa foi aprovado pelo Comitê de Ética em Pesquisa da Universidade Estadual de Ponta Grossa sob número 282.640.

\section{RESULTADOS}

A idade das 190 pacientes idosas hipertensas variou de 60 a 93 anos, com média de 69,5 $\pm 7,4$ anos, sendo que 180 (94,7\%) eram alfabetizadas, $165(86,8 \%)$ moravam com pelo menos mais uma pessoa, $103(54,2 \%)$ não tinham trabalho remunerado, pensão ou aposentadoria e apenas $14(7,4 \%)$ tinham plano de saúde. O tempo de acompanhamento na USF variou de 1 a 26 anos, com média de 13,4 \pm 7,9 anos, e 139 (73,2\%) usavam mais de um medicamento anti-hipertensivo. As variações das pressões arteriais sistólica e diastólica, bem como a média das pressões para as pacientes com PA controlada e não controlada podem ser obervadas na Tabela 1.
$\mathrm{Na}$ distribuição das variáveis sociodemográficas, de acompanhamento clínico, de tratamento e comorbidades entre as pacientes com PA controlada e não controlada foi observada significância estatística para idade superior a 70 anos $(\mathrm{p}=0,03)$ e residir na área mais próxima ou mais distante da USF (Tabela 2).

Para a análise multivariada foram incluídas no modelo as variáveis idade, alfabetização e residir na área mais próxima da USF. A análise de regressão logística demonstrou que as variáveis possuir mais de 70 anos e residir na área mais afastada da USF estavam relacionadas com níveis de PA não controlada (Tabela 3).

\section{DISCUSSÃO}

Foi observado neste estudo que as médias das PAS e das PAD das pacientes idosas hipertensas classificadas como não controladas foram maiores quando comparadas à das pacientes classificadas como controladas (ambos valores de $\mathrm{p}<0,001$ ). $\mathrm{O}$ índice de idosas hipertensas que apresentaram níveis de $\mathrm{PA}$ controlada foi de $56,8 \%$. Esse resultado pode ser considerado bom quando comparado aos dados de uma revisão sobre o controle da HAS em publicações brasileiras até outubro de 2012, a qual encontrou para a população idosa de ambos os sexos taxas que variaram de 27 a 44,6\% ${ }^{4}$. Por exemplo, um estudo de coorte em município de pequeno porte em São Paulo analisou dados dos participantes hipertensos tratados de ambos os sexos com 71-81 anos de idade da linha de base $(n=313)$ e do $11^{\circ}$ ano de seguimento $(\mathrm{n}=484)$. A prevalência do controle adequado da hipertensão $(<140 /<90 \mathrm{mmHg})$ foi semelhante nas duas coortes (44,6\% e 40,1\%), sem diferença significativa, sendo considerado baixo tanto na coorte mais jovem quanto na coorte mais velha ${ }^{16}$.

Estudos também têm sido realizados em outros países sobre controle da PA com idosos hipertensos. Em um Centro de Saúde de Portugal, em uma amostra de 250 idosos em regime ambulatorial foram encontradas para o sexo feminino $(62,4 \%$ da amostra) frequência de controle de $20,4 \%$ para a PAS $(<140 \mathrm{mmHg})$ e de $50,4 \%$ para PAD $(<90 \mathrm{mmHg})^{17}$. Por outro lado, valores maiores de controle da PA em idosos foram descritos em outros países. Em Ontário, Canadá, em 2.551 pessoas pesquisadas, as taxas de controle encontradas foram de 65,7\%. Para os idosos

Tabela 1. Distribuição das pacientes idosas hipertensas acompanhadas em Unidade de Saúde da Família, segundo o controle da pressão arterial $(n=190)$

\begin{tabular}{lccc} 
& PA não controlada & PA controlada & $\boldsymbol{p}^{*}$ \\
N (\%) & $82(43,2)$ & $108(56,8)$ & - \\
PAS mmHg (variação) & 110 a 200 & 80 a 130 & - \\
PAD mmHg (variação) & 60 a 100 & 50 a 80 & - \\
PAS média (desvio-padrão) & $148,7(16,4)$ & $118,9(10,9)$ & $<0,001$ \\
PAD média (desvio-padrão) & $86,9(10,7)$ & $74,0(7,5)$ & $<0,001$ \\
\hline
\end{tabular}

PA: pressão arterial; PAS: pressão arterial sistólica; PAD: pressão arterial diastólica

${ }^{*}$ Mann Whitney Test 
Tabela 2. Distribuição das pacientes idosas hipertensas acompanhadas na USF, segundo o controle da pressão arterial e as variáveis sociodemográficas, de acompanhamento, tratamento e comorbidades $(n=190)$

\begin{tabular}{|c|c|c|c|c|c|c|c|}
\hline \multirow[t]{2}{*}{ Variáveis } & \multicolumn{2}{|c|}{$\begin{array}{l}\text { PA não controlada } \\
\qquad(\mathbf{n}=82)\end{array}$} & \multicolumn{2}{|c|}{$\begin{array}{l}\text { PA controlada } \\
\quad(n=108)\end{array}$} & \multirow{2}{*}{$\begin{array}{c}\text { Razão de } \\
\text { prevalências }\end{array}$} & \multirow[t]{2}{*}{ IC 95\% } & \multirow[t]{2}{*}{$p$} \\
\hline & $\mathbf{n}$ & $(\%)$ & $\mathbf{n}$ & $(\%)$ & & & \\
\hline \multicolumn{8}{|l|}{ Idade em anos } \\
\hline até 70 & 45 & $(54,9)$ & 76 & $(70,4)$ & 1,00 & - & \multirow{2}{*}{0,03} \\
\hline$>70$ anos & 37 & $(45,1)$ & 32 & $(29,6)$ & 1,44 & $1,05-1,98$ & \\
\hline \multicolumn{8}{|l|}{ Alfabetização } \\
\hline Sim & 80 & $(97,6)$ & 100 & $(92,6)$ & 1,00 & - & \multirow{2}{*}{0,19} \\
\hline Não & 2 & $(2,4)$ & 8 & $(7,4)$ & 0,45 & $0,13-1,57$ & \\
\hline \multicolumn{8}{|l|}{ Plano de saúde } \\
\hline Com & 4 & $(4,9)$ & 10 & $(9,3)$ & 1,00 & - & \multirow{2}{*}{0,25} \\
\hline Sem & 78 & $(95,1)$ & 98 & $(90,7)$ & 1,55 & $0,67-3,61$ & \\
\hline \multicolumn{8}{|l|}{ Com quem reside } \\
\hline Com outras pessoas & 72 & $(87,8)$ & 93 & $(86,1)$ & 1,00 & - & \multirow{2}{*}{0,73} \\
\hline Sozinha & 10 & $(12,2)$ & 15 & $(13,9)$ & 0,92 & $0,55-1,53$ & \\
\hline \multicolumn{8}{|l|}{ Trabalho remunerado ${ }^{*}$} \\
\hline Sim & 48 & $(58,5)$ & 55 & $(50,9)$ & 1,00 & - & \multirow{2}{*}{0,30} \\
\hline Não & 34 & $(41,5)$ & 53 & $(49,1)$ & 0,84 & $0,60-1,17$ & \\
\hline \multicolumn{8}{|c|}{ Local da residência em relação à USF } \\
\hline Área A (mais próxima) & 31 & $(37,8)$ & 67 & $(62,0)$ & 1,00 & - & \multirow{2}{*}{0,001} \\
\hline Àrea B (mais afastada) & 51 & $(62,2)$ & 41 & $(38,0)$ & 1,75 & $1,24-2,47$ & \\
\hline \multicolumn{8}{|l|}{ Acompanhamento $>10$ anos } \\
\hline Sim & 36 & $(43,9)$ & 50 & $(46,3)$ & 1,00 & - & \multirow{2}{*}{0,74} \\
\hline Não & 46 & $(56,1)$ & 58 & $(53,7)$ & 1,06 & $0,76-1,47$ & \\
\hline Consultas médicas no últim & & & & & & & \\
\hline Até 2 & 20 & $(24,4)$ & 15 & $(13,9)$ & 1,00 & - & 006 \\
\hline 3 ou mais & 62 & $(75,6)$ & 93 & $(86,1)$ & 1,43 & $1,01-2,02$ & 0,06 \\
\hline Número de anti-hipertensiv & & & & & & & \\
\hline 1 & 25 & $(30,5)$ & 26 & $(24,1)$ & 1,00 & - & 027 \\
\hline 2 ou mais & 57 & $(69,5)$ & 82 & $(75,9)$ & 0,84 & $0,59-1,18$ & 0,32 \\
\hline Diabetes mellitus & & & & & & & \\
\hline Não & 44 & $(53,7)$ & 62 & $(57,4)$ & 1,00 & - & 066 \\
\hline Sim & 38 & $(46,3)$ & 46 & $(42,6)$ & 1,09 & $0,79-1,51$ & 0,01 \\
\hline Dislipidemia & & & & & & & \\
\hline Não & 54 & $(65,9)$ & 63 & $(58,3)$ & 1,00 & - & \\
\hline Sim & 28 & $(34,1)$ & 45 & $(41,7)$ & 0,83 & $0,58-1,18$ & 0,29 \\
\hline Obesidade ${ }^{\star *}$ & & & & & & & \\
\hline Não & 45 & $(57,0)$ & 58 & $(54,2)$ & 1,00 & - & 071 \\
\hline Sim & 34 & $(43,0)$ & 49 & $(45,8)$ & 0,94 & $0,67-1,31$ & 0,71 \\
\hline Doença coronariana & & & & & & & \\
\hline Não & 70 & $(85,4)$ & 94 & $(87,0)$ & 1,00 & - & 074 \\
\hline Sim & 12 & $(14,6)$ & 14 & $(13,0)$ & 1,08 & $0,69-1,70$ & 0,74 \\
\hline Insuficiência cardíaca & & & & & & & \\
\hline Não & 77 & $(93,9)$ & 100 & $(92,6)$ & 1,00 & - & 27 \\
\hline Sim & 5 & $(6,1)$ & 8 & $(7,4)$ & 0,88 & $0,44-1,79$ & 0,72 \\
\hline Acidente vascular cerebral & & & & & & & \\
\hline Não & 79 & $(96,3)$ & 103 & $(95,4)$ & 1,00 & - & 100 \\
\hline Sim & 3 & $(3,7)$ & 5 & $(4,6)$ & 0,86 & $0,35-2,15$ & 1,00 \\
\hline Doença renal crônica & & & & & & & \\
\hline Não & 79 & $(96,3)$ & 107 & $(99,1)$ & 1,00 & - & 027 \\
\hline Sim & 3 & $(3,7)$ & 1 & $(0,9)$ & 1,77 & $0,98-3,19$ & 0,32 \\
\hline
\end{tabular}

*Ou pensão ou aposentadoria; USF: Unidade de Saúde da Família

${ }^{* *}$ Quatro dados faltantes 
Tabela 3. Modelo de regressão logística múltipla para pressão arterial não controlada em idosas hipertensas acompanhadas na USF $(n=190)$

\begin{tabular}{llcc}
\multicolumn{1}{c}{ Variáveis } & OR & $\mathbf{9 5 \% ~ I C ~}$ & $\boldsymbol{p}$ \\
$\begin{array}{l}\text { Idade }>70 \text { anos } \\
\begin{array}{l}\text { Residir na área mais } \\
\text { afastada da USF }\end{array}\end{array}$ & 2,0 & $1,08-3,80$ & 0,033 \\
\hline
\end{tabular}

OR: odds ratio; IC: intervalo de confiança

de ambos os sexos, que representavam 19,6\% da amostra, a taxa de controle $(<140 / 90 \mathrm{mmHg})$ foi de $67,5 \%$. O estudo observa que o manejo da hipertensão aumentou consideravelmente as taxas de tratamento e de controle da doença desde o último levantamento, de 1992, quando as taxas de controle para a população em geral eram de apenas $12,1 \%$. Também ressalta que melhorar a proporção de pacientes controlados ainda é essencial, uma vez que cerca de $30 \%$ de pacientes permaneciam com elevado risco de complicações prematuras cardiovasculares ${ }^{18}$.

Nos Estados Unidos, em estudo utilizando dados do National Health and Nutrition Examination Survey (pesquisa nacional representativa da população norte-americana não institucionalizada) sobre amostra de 2.877 pacientes idosos de ambos os sexos, a prevalência de controle adequado da HAS (PA $<140 / 90 \mathrm{mmHg}$ ) foi de $51,4 \%$. Para a população maior de 18 anos, a maior taxa de controle foi encontrada entre as mulheres, cerca de $55 \%$ em comparação à de $42,7 \%$ entre os homens ${ }^{19}$. Vários estudos indicam que as mulheres tendem a ter maior taxa de controle da PA em relação aos homens. Isso ocorre principalmente porque elas se preocupam mais com sua saúde, em geral, e acompanham melhor a doença nos serviços de saúde, chegando a compor $80 \%$ da demanda desses serviç̧os ${ }^{20-22}$. Esse fato deve ser considerado na análise do percentual de controle da PA encontrado no presente estudo, que possivelmente teria sido menor caso a amostra não fosse composta exclusivamente por mulheres.

O controle da HAS também tem sido pesquisado na Atenção Primária em Saúde para a população adulta, incluindo idosos. Em um estudo brasileiro que aferiu em ambiente ambulatorial a pressão de 135 pacientes, dos quais $84 \%$ eram mulheres, com uma média de idade de 54,9 \pm 9,7 anos, foi observado um índice de $57 \%$ de pacientes com níveis pressóricos adequados (PA < 140/90 mmHg) após implantação do Programa de Saúde da Família, em comparação a 28,9\% no início ${ }^{20}$.

De modo diferente, Hoepfner e Franco ${ }^{5}$ estimaram a prevalência de controle da PA em adultos hipertensos, com média de idade de 61,5 anos, nas Unidades Básicas de Saúde (UBS) de Joinville, durante um ano, observando redução média da PA entre o primeiro e o último registro e prevalência de controle da PA em 36,6\% dos pacientes (PAS $<140 \mathrm{mmHg}$ e PAD $<86 \mathrm{mmHg}$ e $130 / 80 \mathrm{mmHg}$, nos casos de diabetes mellitus e doença renal crônica), resultado considerado baixo pelos autores, que identificaram como possível causa a inércia terapêutica, em que os profissionais de Saúde não realizam mudanças terapêuticas adequadas e/ou os pacientes não aderem a essas mudanças.

Em Petrópolis, no Rio de Janeiro, foram verificadas as taxas de controle da HAS (PA < 140/90 mmHg) de 250 pacientes acompanhados nas USF e comparadas com 250 pacientes acompanhados nas UBS, com média de idade de $36 \pm 14,9$ anos. Foi observado melhor controle da PA nos pacientes acompanhados nas UBS $(39,23 \%)$ quando comparado ao dos pacientes acompanhados nas USF $(29,2 \%)^{23}$.

Apesar da elevada taxa de controle encontrada nesta pesquisa, quando comparada aos dados da literatura em estudos com idosos brasileiros, ainda existe uma grande proporção de pacientes idosas não controladas (43,2\%). Portanto, há necessidade de se buscarem estratégias para aumentar a proporção de pacientes controladas. Com essa finalidade é importante citar-se a capacitação contínua dos médicos, a simplificação nos esquemas medicamentosos e a conscientização dos pacientes sobre a importância do controle de seus níveis pressóricos como mudanças que devem ser implementadas nos três eixos: médico, serviço e paciente. Também importante é o conhecimento do perfil de populações na identificação de fatores de risco específicos, para o desenvolvimento de medidas de saúde pública direcionadas, com o intuito de se obter a maior efetividade possível ${ }^{24}$.

De forma semelhante a outros estudos sobre controle da PA em idosos, não foi observado neste estudo associação significativa entre controle da PA e algumas variáveis analisadas, como obesidade, dislipidemia ${ }^{17}$ e número de consultas médicas no último $\mathrm{ano}^{25}$. Na análise multivariada, observou-se menor taxa de controle da PA para as pacientes com idade superior a 70 anos (OR $=2,0$, IC95\% 1,08-3,80) e para as que moravam na área mais afastada da USF (OR = 2,6, IC95\% 1,42-4,75).

Sabe-se que a prevalência da HAS aumenta nos idosos com idade mais avançada pela tendência do aumento dos níveis pressóricos com o passar dos anos. Estudo realizado em São Paulo evidenciou esse fato. Em uma amostra de 1.265 mulheres idosas, a prevalência da hipertensão aumentou cerca de $5 \%$ entre o grupo de 60 a 74 anos e no grupo de idosas com mais de 75 anos $^{26}$. $\mathrm{O}$ aumento dos níveis pressóricos em idades mais avançadas está possivelmente associado a maior dificuldade na normalização da PA e consequente piora das taxas de controle nessa faixa etária. No estudo Controlar Brasil, realizado em um centro especializado com 2.810 adultos, constatou-se que, para cada ano de vida, a probabilidade de controle da PA foi reduzida em $2 \%{ }^{27}$. Em estudo de coorte já citado, os autores relataram que a maior idade está relacionada a menor comparecimento às consultas médicas e a maior irregularidade no uso de medicamentos ${ }^{16}$.

Dados também sugerem que as taxas de controle da PA são menores em pacientes que comparecem menos às consultas 
na Unidade de Saúde, por estar associado a pior adesão medicamentosa. Uma possível razão para essa correlação é que a adesão medicamentosa depende em grande parte da habilidade de o médico manter uma boa relação com o paciente, enfraquecendo-se o vínculo com pacientes faltosos. Outra razão é a dificuldade nos ajustes posológicos e na introdução de novos fármacos entre pacientes que não comparecem e não utilizam adequadamente a medicação ${ }^{28}$.

Apesar de boa parte dos estudos demonstrarem que um menor comparecimento em consultas médicas pode diminuir a adesão do paciente ao tratamento e prejudicar o seu manejo, neste estudo não foi observada associação entre número de consultas médicas e controle da PA. Contudo, deve-se considerar que a lógica do atendimento em uma USF é da longitudinalidade e integralidade da atenção de equipe interdisciplinar. Nesse sentido, as pacientes podem ser atendidas na USF não apenas na consuta médica mas também por outros profissionais de Saúde e receber orientação em relação ao tratamento e acompanhamento da HAS, o que influenciaria em um melhor controle da doença ${ }^{29}$.

Acredita-se que as pacientes que residem em locais mais afastados da USF possam ter maior dificuldade de acesso ao serviço de saúde, considerando-se, além da dificuldade geográfica, as limitações funcionais próprias da idade. Estudo de revisão norte-americano sobre a epidemiologia do controle inadequado da PA considerou a dificuldade de acesso aos cuidados em saúde como possível causa, dentre outras, de não controle da $\mathrm{PA}^{6}$. Em relação à essa dificuldade, medidas relativas à qualidade do serviço de saúde devem ser consideradas, especialmente quanto aos componentes de estrutura e processo de trabalho ${ }^{30}$, o que muitas vezes vai depender da intervenção da equipe de saúde para viabilizar ou permitir a atenção adequada.

Este estudo apresenta limitações, como a não utilização de dados sobre renda, tabagismo e sedentarismo das pacientes, por não estarem disponíveis nos prontuários. Além disso, por ter sido realizado em apenas uma Unidade de Saúde, os dados não podem ser generalizados. Apesar dessas limitações, pode contribuir para o melhor entendimento do contole da PA em um grupo de pacientes idosas, que representam grande percentual dos usuários dos serviços de saúde.

\section{CONCLUSÃO}

O controle da PA foi observado em $56,8 \%$ das pacientes idosas hipertensas. Embora esse percentual de controle seja elevado quando comparado a estudos brasileiros com idosos hipertensos, medidas devem ser implantadas para o aumento dessa taxa, para evitar futuras complicações cardiovasculares. Atenção especial deve ser dada às pacientes mais idosas e que residem em área mais afastada da USF.

\section{REFERÊNCIAS}

1. Rabetti AC, Freitas SFT. Avaliação das ações em hipertensão arterial sistêmica na atenção básica. Rev Saude Publica. 2011;45(2):258-68. http:// dx.doi.org/10.1590/S0034-89102011005000007. PMid:21344125.

2. Sociedade Brasileira de Cardiologia. Sociedade Brasileira de Hipertensão. Sociedade Brasileira de Nefrologia. VI Diretrizes Brasileiras de Hipertensão. Arq Bras Cardiol. 2010;95(Supl 1):1-51.

3. James PA, Oparil S, Carter BL, Cushman WC, Dennison-Himmelfarb C, Handler J, et al. 2014 evidence-based guideline for the management of high blood pressure in adults: report from the panel members appointed to the Eighth Joint National Committee (JNC 8). JAMA. 2014;311(5):507-20. http://dx.doi.org/10.1001/jama.2013.284427. PMid:24352797.

4. Pinho NA, Pierin AMG. O controle da hipertensão arterial em publicações brasileiras. Arq Bras Cardiol. 2013;101(3):65-73.

5. Hoepfner C, Franco SC. Inércia clínica e controle da hipertensão arterial nas unidades de atenção primária à saúde. Arq Bras Cardiol. 2010;95(2):223-9. http://dx.doi.org/10.1590/S0066-782X2010005000095. PMid:20658090.

6. Wang TJ, Vasan RS. Epidemiology of uncontrolled hypertension in the United States. Circulation. 2005;112(11):1651-62. http://dx.doi.org/10.1161/ CIRCULATIONAHA.104.490599. PMid:16157784.

7. Brasil. Ministério da Saúde. Secretaria de Atenção à Saúde. Departamento de Atenção Básica. Política Nacional de Atenção Básica [Internet]. Brasília; 2012 [citado em 2016 fev. 4]. Disponível em: http://189.28.128.100/dab/ docs/publicacoes/geral/pnab.pdf

8. Brasil. Ministério da Saúde. Secretaria de Vigilância em Saúde. Departamento de Análise de Situação de Saúde. Plano de ações estratégicas para o enfrentamento das doenças crônicas não transmissíveis (DCNT) no Brasil, 2011-2022. Brasília; 2011.

9. Busnello RG, Melchior R, Faccin C, Vettori D, Petter J, Moreira LB, et al. Características associadas ao abandono do acompanhamento de pacientes hipertensos atendidos em um ambulatório de referência. Arq Bras Cardiol. 2001;73(5):349-51. PMid:11359183.

10. Denker MG, Cohen DL. What is an appropriate blood pressure goal for the elderly: review of recent studies and practical recommendations. Clin Interv Aging. 2013;8:1505-17. PMid:24255596.

11. Tomasik T, Gryglewska B, Windak A, Grodzicki T. Hypertension in the elderly: how to treat patients in 2013? Pol Arch Med Wewn. 2013;123(78):409-16. PMid:23828034.

12. Laubscher T, Regier L, Stone S. Hypertension in the elderly: new blood pressure targets and prescribing tips. Can Fam Physician. 2014;60(5):453-6. PMid:24829009.

13. Veronez LL, Simões MJS. Análise da prescrição de medicamentos de pacientes hipertensos atendidos pelo SUS da rede municipal de saúde de Rincão - SP. Rev Ciênc Farm Básica Apl. 2008;29(1):45-51.

14. Longo MAT, Martelli A, Zimmermann AMA. Hipertensão Arterial Sistêmica: aspectos clínicos e análise farmacológica no tratamento dos pacientes de um setor de Psicogeriatria do Instituto Bairral de Psiquiatria, no Município de Itapira, SP. Rev Bras Geriatr Gerontol. 2011;14(2):271-84. http://dx.doi. org/10.1590/S1809-98232011000200008. 
15. Associação Brasileira para o Estudo da Obesidade e da Síndrome Metabólica - ABESO. Diretrizes brasileiras de obesidade. 3. ed. Itapevi: AC Farmacêutica; 2009.

16. Firmo JO, Peixoto SV, Loyola AI Fo, Uchôa E, Lima-Costa MF. Birth cohort differences in hypertension control in a brazilian population of older elderly: the Bambuí cohort study of aging (1997 and 2008). Cad Saude Publica. 2011;27(Supl 3):427-34. http://dx.doi.org/10.1590/S0102311X2011001500013. PMid:21952864.

17. Mártires MAR, Costa MAM, Santos CSV. Obesidade em idosos com hipertensão arterial sistêmica. Texto Contexto Enferm. 2013;22(3):797803. http://dx.doi.org/10.1590/S0104-07072013000300028.

18. Leenen FHH, Dumais J, McInnis NH, Turton P, Stratychuk L, Nemeth $\mathrm{K}$, et al. Results of the Ontario survey on the prevalence and control of hypertension. CMAJ. 2008;178(11):1441-9. http://dx.doi.org/10.1503/ cmaj.071340. PMid:18490640.

19. Keenan NL, Rosendorf KA, Centers for Disease Control and Prevention. Prevalence of hypertension and controlled hypertension-United States, 2005-2008. MMWR Surveill Summ. 2011;(60 Supl 1):94-7.

20. Araujo JC, Guimarães AC. Controle da hipertensão arterial em uma unidade de saúde da família. Rev Saude Publica. 2007;41(3):368-74. http://dx.doi. org/10.1590/S0034-89102007000300007. PMid:17515989.

21. Pereira VOM, Acurcio FA, Guerra AA Jr, Silva GD, Cherchiglia ML. Perfil de utilização de medicamentos por indivíduos com hipertensão arterial e diabetes mellitus em municípios da rede farmácia de Minas. Cad Saude Publica. 2012;28(8):1546-58. http://dx.doi.org/10.1590/S0102311X2012000800013. PMid:22892974.

22. Kanso S, Romero DL, Leite IC, Marques A. A evitabilidade de óbitos entre idosos em São Paulo, Brasil: análise das principais causas de morte. Cad Saude Publica. 2013;29(4):735-48. http://dx.doi.org/10.1590/S0102311X2013000800011. PMid:23568303.

23. Martins TL, Atallah NA, Silva EMK. Blood pressure control in hypertensive patients within Family Health Program versus at Primary Healthcare Units: analytical cross-sectional study. Sao Paulo Med J. 2012;130(3):145-50 http://dx.doi.org/10.1590/S1516-31802012000300003. PMid:22790546.

24. Mancia G, Fagard R, Narkiewicz K, Redón J, Zanchetti A, Böhm M, et al. 2013 ESH/ESC Guidelines for the management of arterial hypertension: the Task Force for the management of arterial hypertension of the European Society of Hypertension (ESH) and of the European Society of Cardiology (ESC). J Hypertens. 2013;31(7):1281-357. http://dx.doi.org/10.1097/01. hjh.0000431740.32696.cc. PMid:23817082.

25. Nations M, Firmo JO, Lima-Costa MF, Uchôa E. Balking blood pressure "control" by older persons of Bambuí, Minas Gerais State, Brazil: an ethnoepidemiological inquiry. Cad Saude Publica. 2011;27(Supl 3):378-S89. http://dx.doi.org/10.1590/S0102-311X2011001500008. PMid:21952859.

26. Oliveira SMJV, Santos JLF, Lebrão ML, Duarte YAO, Pierins AMG, Hipertensão arterial referida em mulheres idosas: prevalência e fatores associados. Rev Text Context Enferm. 2008;17(2):241-9. http://dx.doi. org/10.1590/S0104-07072008000200004.

27. Nobre F, Ribeiro AB, Mion D Jr. Controle da pressão arterial em pacientes sob tratamento anti-hipertensivo no Brasil: Controlar Brasil. Arq Bras Cardiol. 2010;94(5):663-70. http://dx.doi.org/10.1590/S0066-782X2010005000035. PMid:20428719.

28. Coelho EB, Moysés M No, Palhares R, Cardoso MCM, Geleilete TJM, Nobre F. Relação entre a assiduidade às consultas ambulatoriais e o controle da pressão arterial em pacientes hipertensos. Arq Bras Cardiol. 2005;85(3):15761. http://dx.doi.org/10.1590/S0066-782X2005001600002. PMid:16200260.

29. Pires CGS, Mussi FC. Reflecting about assumptions for care in the healthcare education for hypertensive people. Rev Esc Enferm USP. 2009;43(1):229-36. http://dx.doi.org/10.1590/S0080-62342009000100030. PMid:19437877.

30. Novaes HMDN. Avaliação de programas, serviços e tecnologias em saúde. Rev Saude Publica. 2000;34(5):547-9. http://dx.doi.org/10.1590/S003489102000000500018. PMid:11105122.

Recebido em: Fev. 04, 2016

Aprovado em: Mar. 01, 2016 